\title{
On the Cartesian product of non well-covered graphs
}

\author{
Bert L. Hartnell* \\ Department of Mathematics and Computing Science \\ Saint Mary's University \\ Halifax, Nova Scotia, Canada \\ bert.hartnellesmu.ca
}

\author{
Douglas F. Rall ${ }^{\dagger}$ \\ Department of Mathematics \\ Furman University \\ Greenville, SC, U.S.A. \\ doug.rall@furman.edu
}

Submitted: May 1, 2012; Accepted: Apr 21, 2013; Published: Apr 30, 2013

Mathematics Subject Classifications: 05C69, 05C76

\begin{abstract}
A graph is well-covered if every maximal independent set has the same cardinality, namely the vertex independence number. We answer a question of Topp and Volkmann (1992) and prove that if the Cartesian product of two graphs is well-covered, then at least one of them must be well-covered.
\end{abstract}

Keywords: maximal independent set; well-covered; Cartesian product

\section{Introduction}

A well-covered graph $G$ (Plummer [3]) is one in which every maximal independent set of vertices has the same cardinality. That is, every maximal independent set (equivalently, every independent dominating set) is a maximum independent set. This class of graphs has been investigated by many researchers from several different points of view. Among these are attempts to characterize those well-covered graphs with a girth or a maximum degree restriction. For more details on these approaches as well as others see the surveys by Plummer [4] and by Hartnell [2].

Topp and Volkmann [5] investigated how the standard graph products interact with the class of well-covered graphs. They asked the following question that was restated by Fradkin [1].

*Partially supported by NSERC.

†'Supported by a grant from the Simons Foundation (\#209654 to Douglas Rall) and the Herman N. Hipp Endowed Chair. 
Question 1. ([5]) Do there exist non well-covered graphs whose Cartesian product is well-covered?

The principal result of this paper is the following theorem that answers Question 1 in the negative.

Theorem 2. If $G$ and $H$ are graphs whose Cartesian product is well-covered, then at least one of $G$ or $H$ is well-covered.

In Section 2 we define the terms used most often in this paper; standard graph theory terminology is used throughout. We then establish Theorem 2 in Section 3.

\section{Definitions}

If $G_{1}=\left(V_{1}, E_{1}\right)$ and $G_{2}=\left(V_{2}, E_{2}\right)$ are any two graphs, the Cartesian product of $G_{1}$ and $G_{2}$ is the graph denoted $G_{1} \square G_{2}$ whose vertex set is the Cartesian product of their vertex sets $V_{1} \times V_{2}$. Two vertices $\left(x_{1}, x_{2}\right)$ and $\left(y_{1}, y_{2}\right)$ are adjacent in $G_{1} \square G_{2}$ if either $x_{1}=y_{1}$ and $x_{2} y_{2} \in E_{2}$, or $x_{1} y_{1} \in E_{1}$ and $x_{2}=y_{2}$. Note that if $I_{1}$ is independent in $G_{1}$ and $I_{2}$ is independent in $G_{2}$, then the set $I_{1} \times I_{2}$ is independent in $G_{1} \square G_{2}$.

For an arbitrary graph $G$ we follow Fradkin [1] and define a greedy independent decomposition of $G$ to be a partition $A_{1}, A_{2}, \ldots, A_{t}$ of $V(G)$ such that $A_{1}$ is a maximal independent set in $G$, and for each $2 \leqslant i \leqslant t$, the set $A_{i}$ is a maximal independent set in the graph $G-\left(A_{1} \cup \cdots \cup A_{i-1}\right)$. One way to construct maximal independent sets in the Cartesian product $G \square H$ is to select any greedy independent decomposition $A_{1}, A_{2}, \ldots, A_{t}$ of $G$ and an arbitrary greedy independent decomposition $B_{1}, B_{2}, \ldots, B_{s}$ of $H$ and combine them into what is called a "diagonal" set of the product as $M=\cup_{i}\left(A_{i} \times B_{i}\right)$. If $s \neq t$, then there are as many sets in this union as the smaller of $s$ and $t$.

For a vertex $x$ of a graph $G$, the open neighborhood of $x$ is the set $N(x)$ defined by $N(x)=\{w \in V(G) \mid x w \in E(G)\}$, and the closed neighborhood, $N[x]$, of $x$ is the set $N(x) \cup\{x\}$. For $A \subseteq V(G)$ we define $N(A)$ to be $\cup_{x \in A} N(x)$ and $N[A]=N(A) \cup A$. The vertex independence number of a graph $G$ is the cardinality of a largest independent set in $G$. We denote the vertex independence number of $G$ by $\alpha(G)$ and refer to an independent set of this order as an $\alpha(G)$-set. If a graph $G$ has an independent set $M$ such that $G-N[M]=\{x\}$ for some vertex $x$, then $x$ is said to be an isolatable vertex of $G$. The existence of such a vertex is central to our work.

Lemma 3. Let $G$ be a graph in which no vertex is isolatable. If $I$ is any maximum independent set in $G$ and $x$ is any vertex of $I, G-N[I-\{x\}]$ is a clique of order at least two.

Proof. Suppose $I$ is an $\alpha(G)$-set and that $I$ has a vertex $v$ such that the graph $G-N[I-$ $\{v\}]$ has an independent set $A$ of size at least two. Then $I^{\prime}=(I-\{v\}) \cup A$ is independent in $G$ and has order larger than $|I|=\alpha(G)$, a contradiction. Therefore, $G-N[I-\{v\}]$ is a clique. Since $G$ has no isolatable vertex it follows that $G-N[I-\{v\}]$ has order at least two. 


\section{Main Results}

We first reduce the study of when a Cartesian product is well-covered by considering the existence of isolatable vertices in the two factors.

Theorem 4. Suppose that $H$ is not well-covered and $G$ has an isolatable vertex. Then $G \square H$ is not well-covered.

Proof. Let $A$ and $B$ be maximal independent subsets of $H$ with $|A|>|B|$, and suppose that $x$ is an isolatable vertex in $G$. Let $I$ be an independent set in $G$ such that $x$ is an isolated vertex in the graph $G-N[I]$. Extend the independent set $I \times A$ to a maximal independent set $J$ of $(G-N[x]) \square H$. Let $m=|J|$. Note that $J$ dominates $N_{G}(x) \times A$ (and perhaps other vertices of $N_{G}(x) \times V(H)$ ), but $J$ does not contain any vertices from $N_{G}[x] \times V(H)$.

Let $J_{1}=J \cup(\{x\} \times A)$ and $J_{2}=J \cup(\{x\} \times B)$. By the choice of $A$ and $B$ it is clear that $\left|J_{1}\right|>\left|J_{2}\right|$. Let $X_{A}$ denote the set of vertices in $N_{G}(x) \times V(H)$ that are not dominated by $J_{1}$. Similarly, let $X_{B}$ denote the set of vertices in $N_{G}(x) \times V(H)$ that are not dominated by $J_{2}$. Note that any vertex in $N_{G}(x) \times V(H)$ that is dominated by $J_{1}$ is also dominated by $J$. Since $J \subset J_{2}$, it follows that if a vertex of $N_{G}(x) \times V(H)$ is dominated by $J_{1}$ it is also dominated by $J_{2}$. Hence, the set $X_{B}$ is a subset of $X_{A}$.

Choose a maximal independent set $L$ of the subgraph of $G \square H$ induced by $X_{B}$. Then $J_{2} \cup L$ is a maximal independent set in $G \square H$. Extend $L$ to a maximal independent set $M$ of the subgraph of $G \square H$ induced by $X_{A}$. Now, $J_{1} \cup M$ is a maximal independent set of $G \square H$, and

$$
\left|J_{1} \cup M\right|=\left|J_{1}\right|+|M|>\left|J_{2}\right|+|M| \geqslant\left|J_{2}\right|+|L|=\left|J_{2} \cup L\right| .
$$

Therefore, $G \square H$ has maximal independent sets of distinct cardinalities, and thus $G \square H$ is not well-covered.

It now follows that if both of $G$ and $H$ are not well-covered but $G \square H$ is well-covered, then neither $G$ nor $H$ has an isolatable vertex.

Lemma 5. Let $G$ and $H$ be graphs such that neither has an isolatable vertex. If $G \square H$ is well-covered, then both $G$ and $H$ have the property that if $M$ is any maximal independent set of the graph, that graph must have a maximal independent set $N$ that is disjoint from $M$. Furthermore, at least one of $G$ or $H$ has the property that any two disjoint maximal independent sets have the same cardinality.

Proof. As stated above, neither $G$ nor $H$ has an isolatable vertex. Let $I_{1}, I_{2}, \ldots, I_{t}$ be any greedy independent decomposition of $G$ and $J_{1}, J_{2}, \ldots, J_{s}$ be any greedy independent decomposition of $H$. Let $p=\min \{s, t\}$. The (so-called "diagonal") set $M=\left(I_{1} \times J_{1}\right) \cup$ $\left(I_{2} \times J_{2}\right) \cup \cdots \cup\left(I_{p} \times J_{p}\right)$ is maximal independent in $G \square H$. Since $G \square H$ is well-covered, this implies that $\alpha(G \square H)=|M|=\sum_{k=1}^{p}\left|I_{k}\right| \cdot\left|J_{k}\right|$.

Since $J_{1}$ is an independent set in $H$ and $J_{2}$ is a maximal independent set in $H-J_{1}$, if there exists a vertex $u \in J_{1}$ that is not dominated by $J_{2}$, then $u$ is isolated in $H-N\left[J_{2}\right]$. 
(This follows since every neighbor of $u$ in $H$ would thus belong to $V(H)-\left(J_{1} \cup J_{2}\right)$, and none of these remains in $H-N\left[J_{2}\right]$.) This contradicts the fact that $H$ does not have an isolatable vertex. Therefore, $J_{2}$ is actually a maximal independent set in $H$ as well as in $H-J_{1}$. By an identical argument it follows that $I_{2}$ is a maximal independent set in $G$. Suppose that $a=\left|J_{1}\right|$ and $b=\left|J_{2}\right|$ and that $a \neq b$. Let $c=\left|I_{1}\right|$ and $d=\left|I_{2}\right|$. Since $I_{1}$ and $I_{2}$ are disjoint maximal independent sets in $G$, the list $I_{2}, I_{1}, I_{3}, \ldots, I_{t}$ is also a greedy independent decomposition of $G$. This implies

$$
c a+d b+\sum_{k=3}^{p}\left|I_{k}\right| \cdot\left|J_{k}\right|=\alpha(G \square H)=d a+c b+\sum_{k=3}^{p}\left|I_{k}\right| \cdot\left|J_{k}\right|,
$$

since $G \square H$ is well-covered, and thus $c a+d b=d a+c b$. Since $a \neq b$ we get $c=d$; that is, $\left|I_{1}\right|=\left|I_{2}\right|$. Since $I_{1}, I_{2}, \ldots, I_{t}$ is an arbitrary greedy independent decomposition of $G$, the lemma follows.

We now proceed to prove our main result.

Theorem 2. If $G$ and $H$ are graphs such that $G \square H$ is well-covered, then at least one of $G$ or $H$ is well-covered.

Proof. Suppose by way of contradiction that the statement is not true. Let $G$ and $H$ be a pair of graphs neither of which is well-covered but such that $G \square H$ is well-covered. As above we may assume that no vertex of either $G$ or $H$ can be isolated in its own graph. From Lemma 5 we may assume without loss of generality that $G$ has the property that any two maximal independent sets of different cardinalities must intersect nontrivially.

Since $G$ is not well-covered, there exists a maximal independent set whose cardinality is less than $\alpha(G)$. From the collection of all maximal independent sets in $G$ choose a pair, say $I$ and $J$, such that $|J|<|I|=\alpha(G)$ and $|I \cap J|$ is as small as possible. Since $|I| \neq|J|$ there exists $v \in I \cap J$. Let $F=G-N[I-\{v\}]$. By Lemma 3 this subgraph $F$ is a clique of order at least two. Let $w$ be any vertex of $F$ such that $w \neq v$, and let $I^{\prime}=(I-\{v\}) \cup\{w\}$. Note that $I^{\prime}$ is independent, $\left|I^{\prime}\right|=|I|$, and yet $\left|I^{\prime} \cap J\right|=|I \cap J|-1$ contradicting our choice of $I$ and $J$. Therefore, $G$ is well-covered.

\section{References}

[1] A. O. Fradkin. On the well-coveredness of Cartesian products of graphs. Discrete Math., 309:238-246, 2009.

[2] B.L. Hartnell. Well-covered graphs. J. Combin. Math. Combin. Comput., 29:107-115, 1999.

[3] M.D. Plummer. Some covering concepts in graphs. J. Combin. Theory, 8:91-98, 1970.

[4] M. D. Plummer. Well-covered graphs: a survey. Quaestiones Math., 16(3):253-287, 1993.

[5] J. Topp and L. Volkmann. On the well coveredness of products of graphs. Ars Combin., 33:199-215, 1992. 